
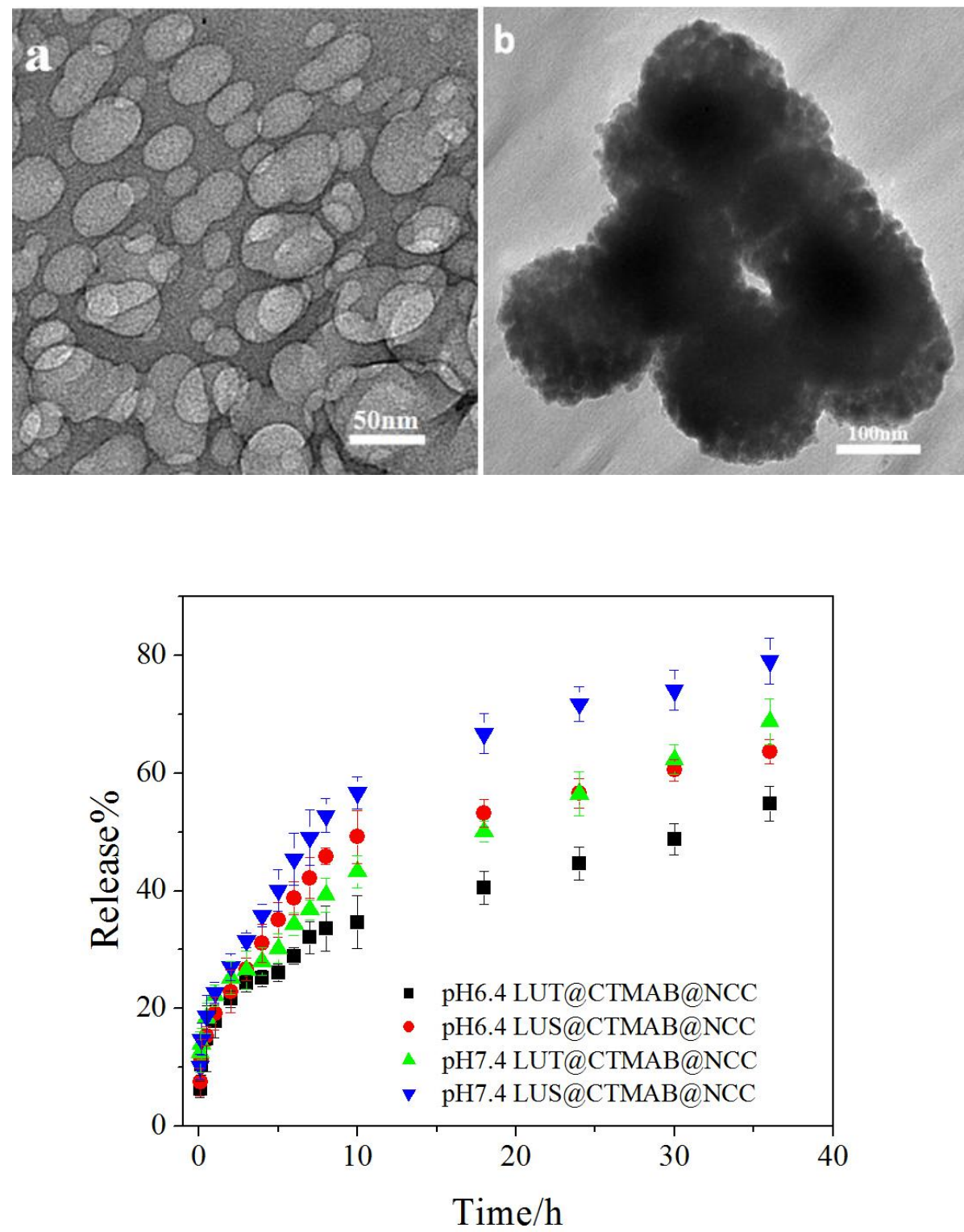


\title{
The Modified Nanocrystalline Cellulose for Hydrophobic Drug Delivery
}

Weixia Qing ${ }^{1,2}$, Yong Wang ${ }^{1}$, Youyou Wang ${ }^{1,3}$, Dongbao Zhao ${ }^{1}$, Xiuhua Liu ${ }^{1,3 *}$, Jinhua Zhu ${ }^{1}$

${ }^{1}$ Institute of Environmental and Analytical Sciences, College of Chemistry and Chemical Engineering, Henan University, Kaifeng, 475004, P.R. China.

2 Medical College, Henan University, Kaifeng, 475004, P.R. China.

${ }^{3}$ Key Lab of Natural Medicine and Immun-engineering of Henan Province, Henan University, Kaifeng, 475004, P.R. China.

\begin{abstract}
In this work, torispherical Nanocrystalline cellulose (NCC) was synthesized, and firstly modified with a cationic surfactant cetyltrimethylammonium bromide (CTMAB). It was proved that the kinetics of NCC adsorbing CTMAB followed the pseudo-second-order kinetics equation, and the adsorption isotherm model followed Freundlich which was multi molecular layer adsorption model. The morphology and structure of NCC and CTMAB-coated NCC were characterized by transmission electron microscopy (TEM) and X-ray powder diffraction (XRD). Stabilities of NCC and CTMAB-coated NCC were assayed by zeta potential. The results showed that NCC in CTMAB solution was well-dispersed and stable. Moreover, the drug loading and release performance of CTMAB-coated NCC were studied using luteolin (LUT) and luteoloside (LUS) as model drugs.
\end{abstract}

Keywords: Nanocrystalline cellulose (NCC), cationic surfactant cetyltrimethylammonium bromide (CTMAB), luteolin (LUT), luteoloside (LUS), drug delivery

* Corresponding author Tel: +86-371-22199505, Email: 11514527@163.com 


\section{Introduction}

Luteolin (LUT, 3', 4', 5, 7-tetrahydroxyflavone, Fig. 1a) is a class of compounds known as flavonoids which are generally characterized by polyphenol chemical structures derived from benzopyrone. LUT mostly exists in various fruits, vegetables and medical plants. It has been illustrated that LUT has numerous biological and pharmacological activities, including antitumorigenic, ${ }^{[1-9]}$ anti-inflammatory, ${ }^{[10-13]}$ anti-allergic, ${ }^{[13,14]}$ anti-amnesic activities, ${ }^{[15]}$ etc. Luteoloside (LUS, 3', 4', 5-trihydroxyflavone-7-O- $\beta$-glucoside, Fig. 1b) is the glucoside of LUT with a $7-\mathrm{O}-\beta$-glucose moiety in the molecular structure, which is abundant in the honeysuckle and dandelion flower. Several studies have revealed that LUS has anti-inflammatory, anticancer, anti-ischemic and anti-HBV effects ${ }^{[10,12,16-18]}$. Although both compounds have great medicinal potentiality, they still have some practical difficulties in applications, such as water-insolubility, low bioavailability and local toxicity. ${ }^{[19,20]}$ In order to overcome these issues, potential drug delivery systems need to be formulated urgently.
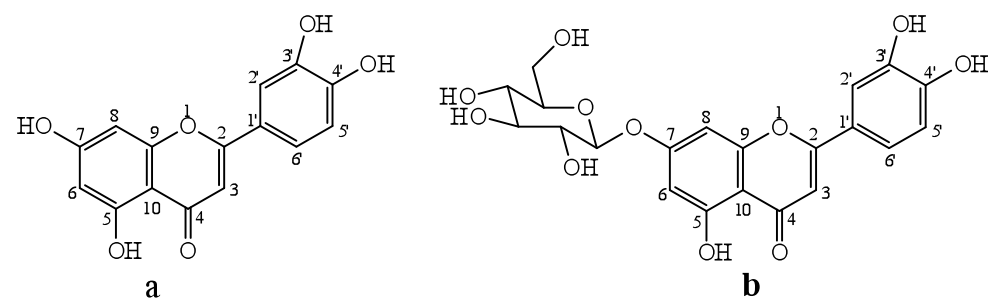

Fig. 1. The chemical structural formula of LUT (a) and LUS (b)

Nanocrystalline cellulose (NCC) was actively investigated as a nanocomposite material, ${ }^{[21-22]}$, since Ränby reported that colloidal suspensions of cellulose can be obtained by controlled sulfuric acid-catalyzed degradation of cellulose fibers ${ }^{[23]}$. So far, NCC has been extensively explored in biomedical fields as a biomaterial, because of biocompatibility, biodegradablity and low cytotoxicity ${ }^{[24-26]}$. According to previous studies, NCC is mostly rod-shaped, ${ }^{[27-28]}$ and allows for prolonged circulation in the bloodstream ${ }^{[29]}$. Therefore, it is mainly used in tissue engineering ${ }^{[30-34]}$, and drug delivery systems ${ }^{[35]}$. However, the drug loading capability of the natural cellulose is very low, so the chemical modification of the natural cellulose is important to improve its practical use for drug carrier ${ }^{[36-38]}$. It may improve the bioavailability of the drug by the surface modification of $\mathrm{NCC}^{[39]}$.

In this paper, on the basis of modified literature, NCC was synthesized by sulfuric acid hydrolysis. It is interesting that the morphology of synthetic NCC is torispherical, which is more 
suitable for drug delivery for its huge specific surface area. In order to improve the drug loading capability, the surface of NCC was modified with a cationic surfactant cetyltrimethyl ammonium bromide (CTMAB), because of the anionic sulfate groups on the surface of $\mathrm{NCC}^{[40]}$. Herein, the morphology and structure of the CTMAB-coated NCC were characterized by transmission electron microscopy (TEM) and X-ray powder diffraction (XRD). Here, CTMABcoated NCC was applied in drug delivery, and the drug loading and release performance of CTMAB-coated NCC were studied using LUT and LUS as model drugs.

\section{Experimental}

\subsection{Chemicals and Materials}

All chemicals were of analytical grade and supplied by Sinopharm Chemical Reagent Co., Ltd (Shanghai, China). Microcryatalline cellulose (MCC) and LUT were purchased from SigmaAldrich. LUS was isolated and prepared from crude extract of Lonicera japonica leaves by highspeed countercurrent chromatography (HSCCC). The purity was $98.0 \%$ which was determined by HPLC and calculated with peak area normalization method. The water used in the experiments was double distilled water.

\subsection{Preparation of $N C C$}

According to the modified method of Dong ${ }^{[41]}$, suspension of NCC was synthesized by acid hydrolysis of MCC. MCC (5 g) was first treated with $60 \mathrm{~mL}$ of sulfuric acid solution $(64 \%$, $\mathrm{wt} \%$ ) at $45^{\circ} \mathrm{C}$ for 30 min under constant magnetic stirring. Then suspension was continued to hydrolyze under ultrasonic treatment at $50 \mathrm{~Hz}$ for $10 \mathrm{~min}$. Then reaction was terminated by adding $600 \mathrm{~mL}$ deionized water, subsequently, the suspension was centrifuged and washed for $4 \sim 5$ times. Next, the suspension had been dialyzed until $\mathrm{pH}$ value of the dialysate was $6 \sim 7$. In the end, the NCC suspension was dried by freezing.

\subsection{Preparation of the drug carrier (CTMAB@NCC)}

Under the optimized conditions, NCC $(0.05 \mathrm{~g})$ was fully reacted with certain concentration of CTMAB. After the suspension was repeatedly washed and centrifuged, a novel drug carrier labeled with $\mathrm{CTMAB} @ \mathrm{NCC}$ was collected by freeze drying.

\subsection{Characterization}

A few drops solution of NCC and CTMAB@NCC were respectively placed on carbon-coated copper grids (200 mesh) and natural dried. Then the morphologies of samples were studied on a JEM-2100 (JEOL Co., Japan). X-Ray diffraction (XRD) patterns were recorded on a Bruker D8 
Advance diffractometer with $\mathrm{Cu} \mathrm{K} \alpha$ radiation and $2 \theta$ range was $10-60^{\circ}$. The zeta potential of samples was recorded on a Nano ZS 90 at $25^{\circ} \mathrm{C}$. Thermogravimetric analyses were recorded using a Mettler-Toledo DTA/TGA instrument in the temperature range from $25-500{ }^{\circ} \mathrm{C}$ at a rate of $10^{\circ} \mathrm{C} \mathrm{min}{ }^{-1}$ under nitrogen flow. The FTIR spectra of samples were recorded on a Thermo Nicolet Avatar 360 spectrometer in transmittance mode by the use of $\mathrm{KBr}$ pellets. Measurements were taken in the wavelength ranging from $500 \mathrm{~cm}^{-1}$ to $4000 \mathrm{~cm}^{-1}$.

\subsection{Study on CTMAB-coated NCC}

CTMAB-coated NCC was carried out by shaking a certain amount of NCC with $25 \mathrm{~mL}$ of the CTMAB solution in different conical flasks using a temperature controlled shaker at $140 \mathrm{rpm}$ for some time. Then, the mixtures were separated and supernatant fluid was analyzed for its residual concentration. The residual concentration of CTMAB was determined by the color-reaction of CTMAB with methyl orange in phosphate buffer (pH6.6), and the absorbance was recorded on UV-Vis spectrophotometer at $470 \mathrm{~nm}$. The amount of CTMAB-coated NCC $\left(q, \mathrm{mg} \cdot \mathrm{g}^{-1}\right)$ was calculated from the equation 1 as:

$$
q=\frac{C_{0}-C_{e}}{m} \times V
$$

Where $C_{0}$ and $C_{\mathrm{e}}\left(\mathrm{mg} \cdot \mathrm{mL}^{-1}\right)$ are initial and equilibrium concentrations of CTMAB in solution, respectively. $m$ is the weight of $\mathrm{NCC}(\mathrm{g}), V$ is the volume of solution (L).

\subsection{Loading of LUT and LUS}

CTMAB@NCC (5 mg) was fully dispersed in double distilled water. Then $200 \mu \mathrm{L}$ of LUT (or LUS) solution $\left(0.21 \mathrm{mg} \cdot \mathrm{mL}^{-1}\right)$ in ethanol and water $(v: v=4: 1)$ was drop by drop added to CTMAB@NCC solution under vigorous stirring. The suspension was incubated at proper temperature under stirring for $30 \mathrm{~min}$, and high-speed centrifuged for $10 \mathrm{~min}$. The amount of unbound drug in the supernatant was determined by UV-Vis spectrophotometer. Flocculent precipitates were dried by freeze drying, which were labeled with LUT@CTMAB@NCC (or LUS@CTMAB@NCC), and reserved. The drug loading capacity was expressed using equation1.

\subsection{In vitro drug release}

LUT@CTMAB@NCC and LUS@CTMAB@NCC were released in a sustained manner in physiological condition mimicked by PBS buffer of $\mathrm{pH} 6.4$ and 7.4 at $37^{\circ} \mathrm{C}$. At predetermined time, the suspension was high-speed centrifuged for $1 \mathrm{~min}$, and the supernatant was removed for 
drug quantification by UV-Vis for LUT and LUS, as previously described. At each sampling time point, fresh release medium was added to the test tube, and the drug delivery system was resuspended. The accumulative percentage release $(Q \%)$ was calculated from the equation 2 as:

$$
Q \%=\frac{C_{n} \times V_{0}+V_{i} \sum_{i=1}^{n-1} C_{i}}{m} \times 100
$$

Where $C_{n}\left(\mathrm{mg} \cdot \mathrm{mL}^{-1}\right)$ is the concentration of drug in the sample, $V_{0}(\mathrm{~mL})$ is the volume of the release medium, $V_{i}(\mathrm{~mL})$ is the volume of the replaced medium, $m(\mathrm{mg})$ is the amount of drug in the sample.

\section{Results and discussion}

\subsection{Characterization of NCC and CTMAB@NCC}

Fig. 2 showed the TEM images of NCC and CTMAB@NCC, respectively. It is clear that the NCC particles are approximately $50 \mathrm{~nm}$ in diameter with nearly spherical morphology. The modified NCC exhibit obviously thicker diameters of nearby $150 \mathrm{~nm}$ with relatively good dispersions compared with precursor NCC.
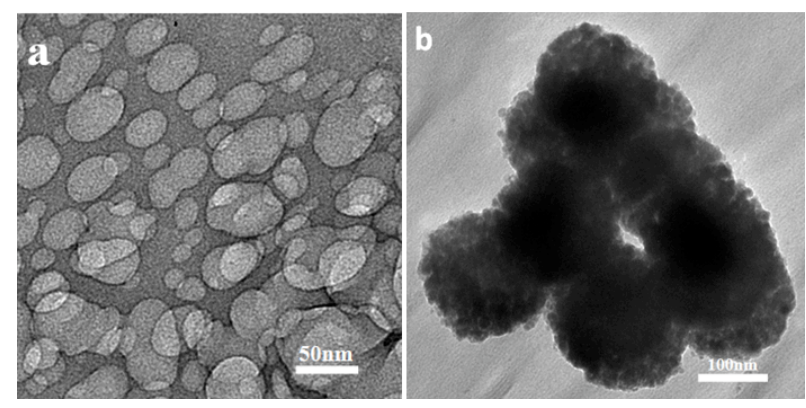

Fig. 2. TEM of NCC (a) and CTMAB@NCC (b)

As shown in Fig. 3a, the X-ray powder diffraction pattern of MCC, NCC and CTMAB@NCC are similar, showing four diffraction peaks, which correspond to the (1-10), (110), (200) and (004) crystallographic planes of cellulose I, respectively. . The crystallinity index $(\mathrm{Crl})$ of the samples were calculated to be $57.1 \%, 70.2 \%$ and $67.3 \%$, respectively, using peak intensity method with following equation: $C r l=\frac{I_{200}-I_{a m}}{I_{200}}$, where the $I_{200}$ is the intensity for the crystalline portion near $2 \theta=22^{\circ}$, and $I_{\mathrm{am}}$ is the intensity for the amorphous portion at about $2 \theta=16^{\circ}$. It is clear that the crystalline peak area increases greatly after sulfuric acid hydrolysis. NCC and 
CTMAB@NCC crystals are more complete, compared with precursor MCC, and chemical modification can't significantly affect the crystal lattice.
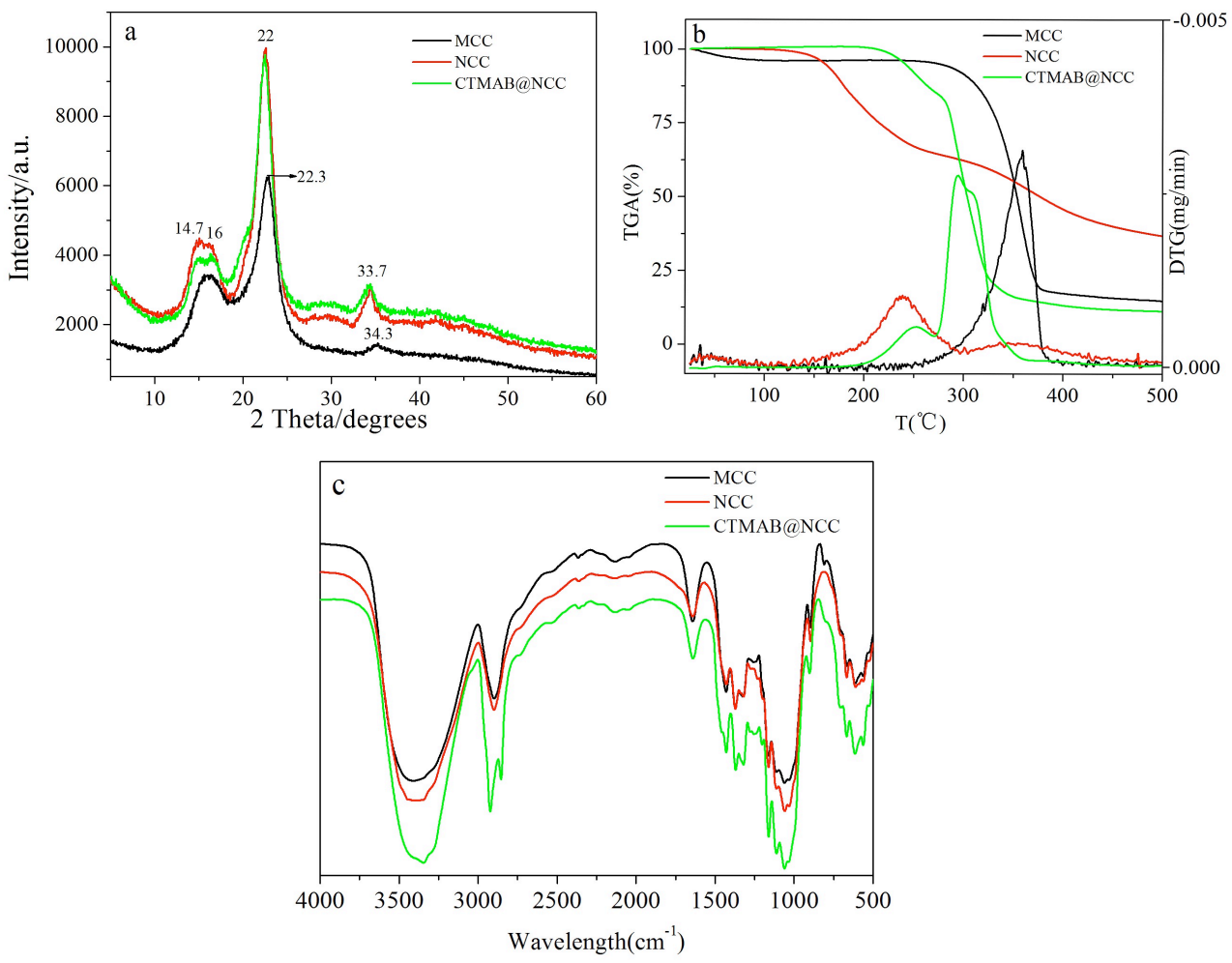

Fig. 3. X-ray powder diffraction (a), TGA and DTG curves (b) and FTIR spectra (c) of MCC, NCC and CTMAB@NCC

Thermogravimetric (TG) and thermogravimetric (DTG) curves were exhibited in Fig. 3b. An initial weight loss at a temperature below $150^{\circ} \mathrm{C}$ is observed due to the loss of moisture present on the surface for all the samples. In the higher temperature range, the thermal decomposition temperatures of $\mathrm{NCC}$ and $\mathrm{CTMAB} @ \mathrm{NCC}$ are lower than that of $\mathrm{MCC}$, which may be attributed to the thermal cleavage of the organic material and the scission of the glucosidic units.

As shown in Fig. 3c, the FTIR spectra of samples are same similar. Signals at about $3350 \mathrm{~cm}^{-1}$ corresponds to $-\mathrm{O}-\mathrm{H}$ vibration, the band between 2800 and $3000 \mathrm{~cm}^{-1}$ corresponds to asymmetric and symmetric $\mathrm{C}-\mathrm{H}$ stretching vibration, and the peak at about $1460 \mathrm{~cm}^{-1}$ corresponds to $-\mathrm{C}-\mathrm{N}$ stretching vibration. Meanwhile, the peaks present at about 1430, 1163 and $1110 \mathrm{~cm}^{-1}$ are assigned to $-\mathrm{CH}_{2}$ symmetric bending, asymmetric $\mathrm{C}-\mathrm{O}-\mathrm{C}$ bridge stretching, anhydroglucose ring asymmetric stretching, respectively, being associated with the crystalline part of the cellulose. Moreover, the peak at about $900 \mathrm{~cm}^{-1}$ is assigned to the vibration frequency of cellulose 
anomeric carbon. In general, characteristic peaks on the spectra have no obvious change, demonstrating that NCC and NCC@CTMAB are still the basic chemical structure of cellulose.

As shown in Fig. 4, NCC was a negative charge in water as evidenced by a zeta potential of approximately $-34.2 \mathrm{mv}$. However, after modified with CTMAB, the zeta potential of NCC was rapidly increased to $21.5 \mathrm{mv}$. Obviously, there are chemical bond between NCC and CTMAB, mainly including electrostatic force and hydrogen bond, and the two suspensions are relatively stable.

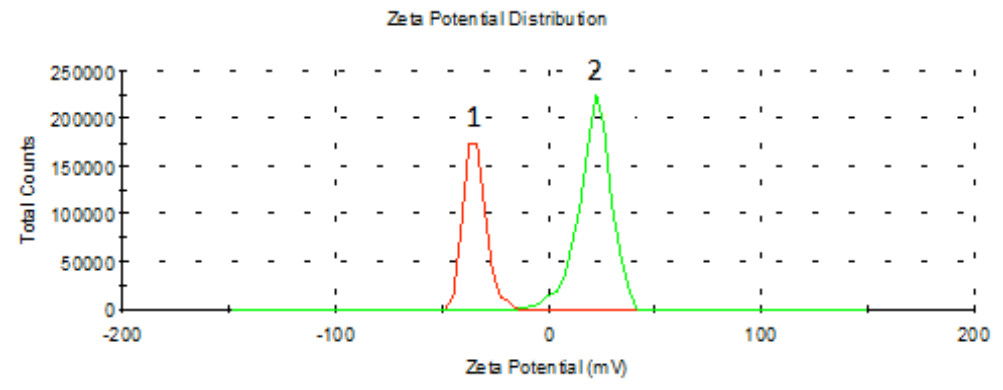

Fig. 4. Zeta potential of NCC (1) and CTMAB@NCC (2)

\subsection{Study on CTMAB-coated NCC}

\subsubsection{Effect of pH, temperature and adsorption time}

The $\mathrm{pH}$ range of 4 to 9 adjusted by $0.1 \mathrm{~mol} \cdot \mathrm{L}^{-1} \mathrm{HCl}$ or $\mathrm{NaOH}$ solution was selected to study the NCC loading capacities of CTMAB and the results were presented in Fig. 5a. When pH was about 6.9 , the adsorption quantity was the largest. This experiment phenomenon is related to the surface charge density of NCC, because the positively charged CTMAB will generate static electrostatic interaction with the negatively charged NCC. The change of adsorption capacity with the increase of temperature was recorded within the range of $15^{-} 65^{\circ} \mathrm{C}$. It was found that the adsorption capacity of NCC increased slowly until up to $40^{\circ} \mathrm{C}$ (Fig. 5b). When shaking time was changed, as shown in Fig. 5c, the adsorption reached equilibrium within no more than 30 min.
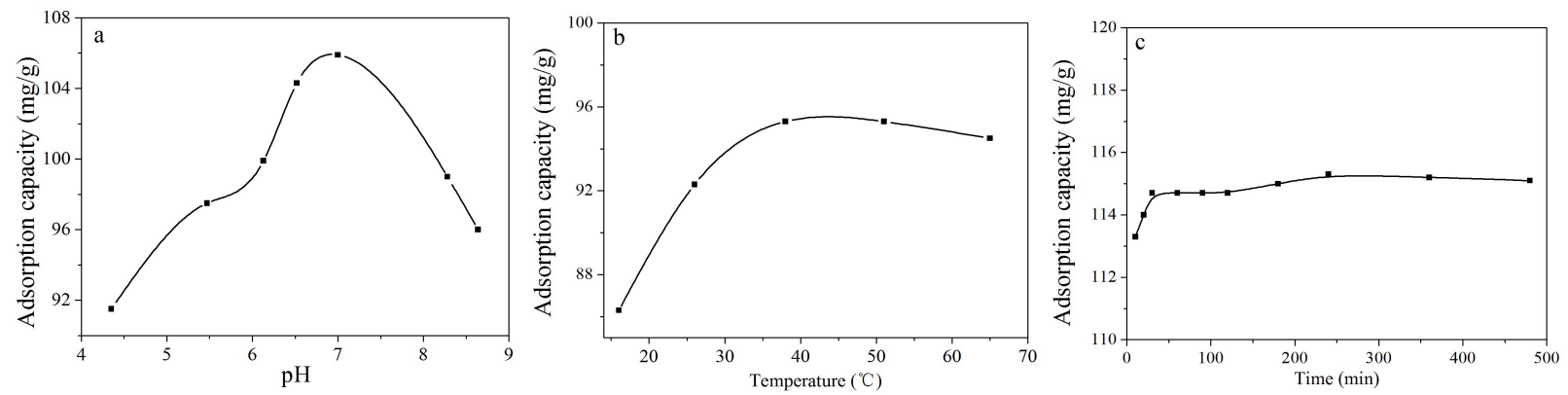

Fig. 5. Effect of pH (a), temperature (b) and shaking time (c) on adsorption 


\subsubsection{Adsorption kinetics studies}

The adsorption kinetics was studied to manifest the adsorption rate of CTMAB onto NCC as well as the rate controlled equilibrium time. The experimental data was analyzed using the pseudo-second-order equation (equation 3 ) as follows:

$$
\frac{t}{q_{t}}=\frac{1}{k_{2} q_{e}^{2}}+\frac{1}{q_{e}} t
$$

Where $q_{\mathrm{e}}\left(\mathrm{mg} \cdot \mathrm{g}^{-1}\right)$ is the equilibrium adsorption capacity, $q_{\mathrm{t}}\left(\mathrm{mg} \cdot \mathrm{g}^{-1}\right)$ is the adsorption capacity at any time $\mathrm{t}, k_{2}\left(\mathrm{dm}^{3} \cdot \mathrm{mg}^{-1} \cdot \mathrm{min}^{-1}\right)$ is the rate constant of pseudo-second-order adsorption.

The experimental data and the linear fitting were shown in Fig. 6. The correlation coefficient and some other important parameters obtained from the fitting were presented in table1. The plot appeared in good linearity with a high correlation coefficient $\left(\mathrm{R}^{2}=0.9999\right)$, and the theoretical $q_{\mathrm{e}}$ value $\left(115.21 \mathrm{mg} \cdot \mathrm{g}^{-1}\right)$ was very close to the experimental data $\left(115 \mathrm{mg} \cdot \mathrm{g}^{-1}\right)$. These results indicated the pseudo-second-order kinetics model described quite well the experiment data.

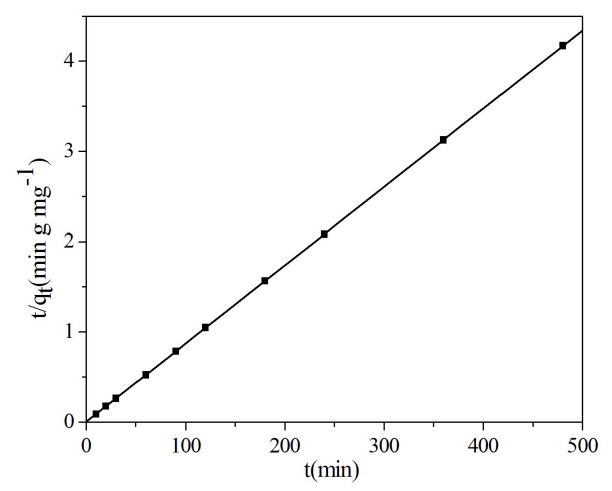

Fig. 6. The linear fitting of pseudo-second-order

Table1. Adsorption kinetics constants for the adsorption of CTMAB onto NCC

Sample

CTMAB@NCC

Pseudo-second-order adsorption kinetics model

\begin{tabular}{ccc}
$K_{2}\left(\mathrm{~g} \cdot \mathrm{mg}^{-1} \cdot \mathrm{min}^{-1}\right)$ & $q_{\mathrm{e}}\left(\mathrm{mg} \cdot \mathrm{g}^{-1}\right)$ & $R^{2}$ \\
\hline 0.03675 & 115.21 & 0.9999
\end{tabular}

\subsubsection{Adsorption isotherms}

As shown in Fig. 7a, adsorption isotherm reach platform, when the equilibrium concentration of CTMAB was about $1.17 \times 10^{-3} \mathrm{~mol} \cdot \mathrm{L}^{-1}$. However, the adsorption capacity increased with increasing $C_{\mathrm{NaCl}}$ (Fig. 7b). The reason is related to ionic strength. With increasing concentration of $\mathrm{NaCl}$, electrostatic repulsion between molecules also increases, which can change the adsorption configuration, and further improve adsorption capacity. Moreover, it is related to 
solubility of CTMAB, and CTMAB is more likely to be adsorbed in the high concentration solution of $\mathrm{NaCl}$.
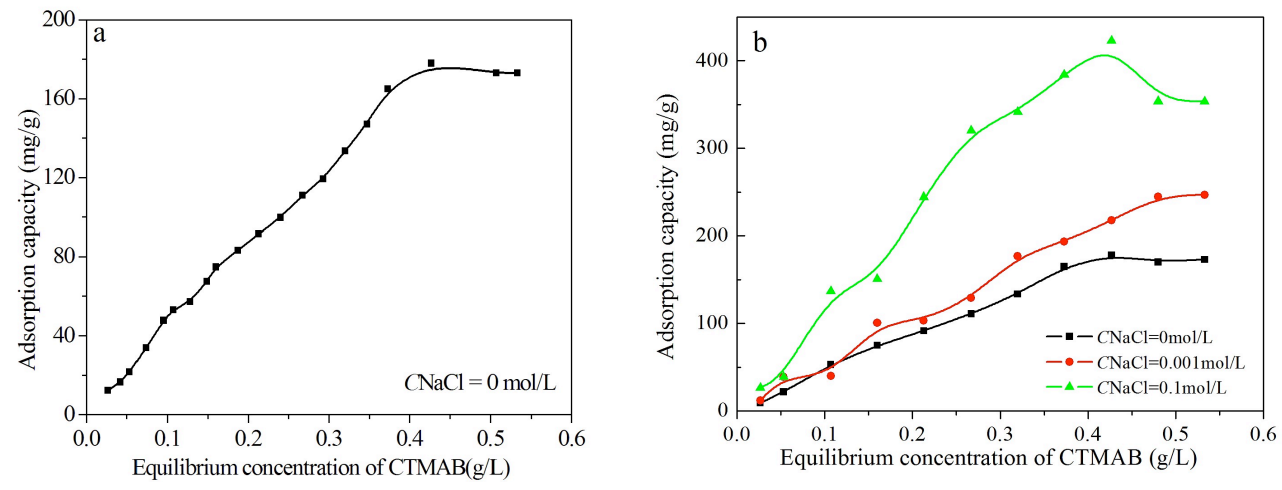

Fig. 7 The adsorption isotherm of CTMAB with different $C_{\mathrm{NaCl}}$

The adsorption isotherm models of Langmuir and Freundlich can be expressed as in the linear form of equation 4 and 5, respectively. The parameters calculated from the two models were presented in table 2, Fig. 8 showed the linear plots of adsorption isotherm for the CTMAB adsorption on NCC at the optimum experiment conditions. Compared with Langmuir isotherm, Freundlich isotherm could better describe the adsorption behaviors with a correlation coefficient $\mathrm{R}^{2}>0.95$, indicating a multi molecular layer adsorption of CTMAB onto NCC surface.

$$
\begin{gathered}
\frac{c_{e}}{q_{e}}=\frac{c_{e}}{q_{m}}+\frac{1}{K_{L} q_{m}} \\
\log q_{e}=\log K_{F}+\log \frac{c_{e}}{n_{F}}
\end{gathered}
$$

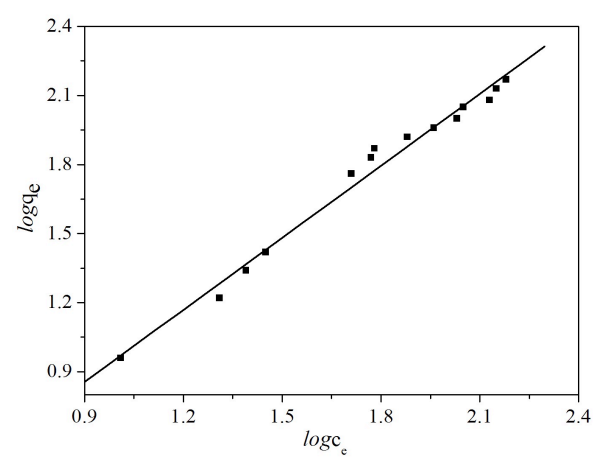

Fig. 8. The linear fitting of Freundich model

Table2. The isotherm models for adsorption of adsorbate

\begin{tabular}{cccccccc}
\hline Sample & \multicolumn{3}{c}{ Langmuir model } & \multicolumn{4}{c}{ Freundich model } \\
& $q_{\mathrm{m}}(\mathrm{mg} / \mathrm{g})$ & $K_{\mathrm{L}}(\mathrm{L} / \mathrm{mg})$ & $R^{2}$ & & $n_{\mathrm{F}}$ & $K_{\mathrm{F}}(\mathrm{L} / \mathrm{mg})$ & $R^{2}$ \\
\hline CTMAB@NCC & 442.48 & 2.99 & 0.8748 & 0.96 & 825.09 & 0.992 \\
\hline
\end{tabular}




\subsection{Loading of LUT and LUS}

It is possible to create a hydrophobic domain on the surface of NCC by coating the negatively charged NCC with CTMAB, and hydrophobic drugs LUT and LUS partitioned strongly into the CTMAB domains on NCC using free drug solutions at low concentrations. A clear charge interaction between positively charged CTMAB@NCC and negatively charged LUT and LUS, was observed, because the positive charge transformed into negative charge (Fig. 9). Obviously, CTMAB was the cross-linker between NCC and flavonoids. The loading content of LUT and LUS are $12.9 \pm 1.5$ and $56.9 \pm 0.9 \mathrm{mg} / \mathrm{g}$, respectively. The two drug delivery systems both had negative charges in water as evidenced by zeta potentials of approximately -28.7 and $-32.3 \mathrm{mV}$, respectively, indicating that the drug complexes are relatively stable.

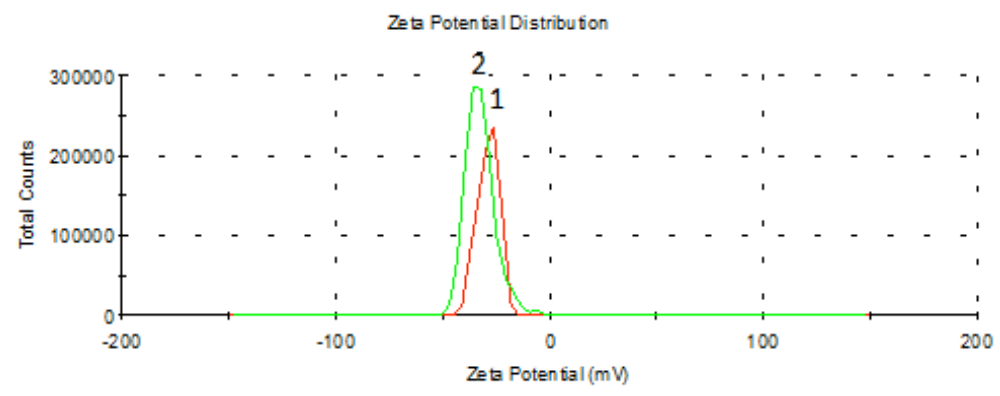

Fig.9. Zeta potential of LUT@CTMAB@NCC (1) and LUS@CTMAB@NCC (2)

\subsection{Release of the drug delivery system}

The drug content was analyzed by UV-Vis at $351 \mathrm{~nm}$ and the assay method was validated. The calibration curves of LUT and LUS were $\mathrm{A}=-0.0084+65.97 C_{\mathrm{LUT}}\left(\mathrm{R}^{2}=0.9990\right)$ and $\mathrm{A}=$ $0.0014+54.45 C_{\text {LUS }}\left(\mathrm{R}^{2}=0.9998\right)$, respectively. Fig. 10 showed the release profiles of LUT@CTMAB@NCC and LUS@CTMAB@NCC. The amount of the two drug complexes released at $\mathrm{pH} 7.4$ is higher than those delivered in $\mathrm{pH}$ 6.4. The cumulative drug release percentage from LUT@CTMAB@NCC was found sustained over a period of $24 \mathrm{~h}$. At pH of 6.4, only $44 \%$ of drug was released, whereas at $\mathrm{pH} 7.4,57 \%$ of the drug was released. For LUS@CTMAB@NCC, at of pH 6.4, 57 \% of drug was released, whereas at pH 7.4, 72\% of the drug was released. The reason is mainly ascribed to two factors. First, the weak acidic drugs can easily ionized under the weak alkaline. Second, the dissociation constants of the drugs show obvious effect. According to the literatures, dissociation constants pKa of LUT are 10.3 and 10.8 ${ }^{[42-43]}$, The dissociation constants pKa of LUS are calculated to be 7.63 and 10.37 using ThamerVoigt equation by our laboratory. It is clear that pKa of LUS is closer to that of the release 
solution. So the release amount and rate of LUS@CTMAB@NCC in both two pH solutions are higher.

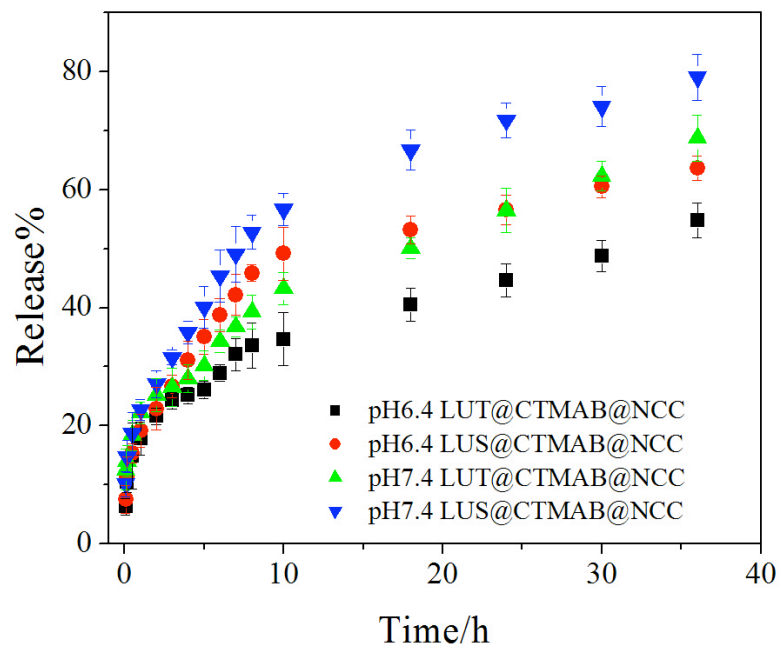

Fig. 10. Release of LUT@CTMAB@NCC and LUS@CTMAB@NCC at 37 C using PBS buffer

\section{Conclusion} solution

In this work, torispherical NCC was synthesized and modified by binding CTMAB. It was proved that the kinetics of NCC adsorbing CTMAB followed the pseudo-second-order kinetics equation, and the adsorption isotherm model followed Freundlich which was multi molecular layer adsorption model. CTMAB-coated NCC was shown to bind amount of the water-insoluble anticancer drugs LUT and LUS. In addition, the conjugates of LUT and LUS with NCC form stable suspension solution. Both LUT and LUS release were found sustained over one day and the results were relatively satisfactory. Overall, this method established a novel drug delivery system which is potential to modulate the loading and release of drugs.

\section{Acknowledgment}

This work was supported by the Support Plan of Science and Technology Innovation team in Universities and Colleges in Henan Province of China (No.14IRTSTHN030), Key Project of Science and Technology Research in Education Department of Henan Province in China (No.14A150011), Key Technology Research Program of Henan Province in China (No. 152102210257) and National Science Foundation of China (U1204304). 


\section{References}

[1] A.K. Pandurangan, P. Dharmalingam, S. Kumar Ananda Sadagopan, S. Ganapasam, Asian Pac. J. Cancer Prev. 13(2012)1569.

[2] L.M. Wang, K.P. Xie, H.N. Huo, F. Shang, W. Zou, M.J. Xie, Asian Pac. J. Cancer Prev. 13 (2012) 1431.

[3] F. Xie, Q. Lang, M. Zhou, H. Zhang, Z. Zhang, Y. Zhang, B. Wan, Q. Huang, L. Yu, Eur. J. Pharm. Sci. 46 (2012) 388.

[4] S.H. Park, H.S. Park, J.H. Lee, G.Y. Chi, G.Y. Kim, S.K. Moon, Y.C. Chang, J.W. Hyun, Y.H. Choi, Food Chem. Toxicol. 56 (2013) 100.

[5] P. Pratheeshkumar, Y. Son, A. Budhraja, X. Wang, S. Ding, L. Wang, A. Hitron, J. Lee, D. Kim, S.P. Divya, G. Chen, Z. Zhang, J. Luo, X. Shi, PLOS ONE 7 (2012) e52279.

[6] S. Attoub, A.H. Hassan, B. Vanhoecke, R. Iratni, T. Takahashi, A.M. Gaben, M. Bracke, S. Awad, A. John, H.A. Kamalboor, M.A.A. Sultan, K. Arafat, C. Gespach, G. Petroianu, Eur. J. Pharmcol. 651 (2011) 18.

[7] Y. Jeon, Y.J. Suh, Oncol. Rep. 29 (2013) 819.

[8] Q. Zhang, L. Wan, Y. Guo, N. Cheng, W. Cheng, Q. Sun, J. Zhu, J. Biol. Regul. Homeost. Agents 23 (2009) 71.

[9] Y. Lim, Y. Jeong, A.L. Tyner, J.H. Park, Am. J. Physiol. Gastrointest. Liver Physiol. 292 (2007) G66.

[10] G. Odontuya, J.R. Hoult, P.J. Houghton, Phytother. Res. 19 (2005) 782.

[11] C. Chen, W. Peng, K.Tsai, S. Hsu, Life Sci. 81 (2007) 1602.

[12] C. Hu, D.D. Kitts, Mol. Cell. Biochem. 265 (2004) 107.

[13] H. Veda, C. Yamazaki, M. Yamazaki, Biol. Pharm. Bull. 25 (2002) 1197.

[14] M. Kimata, N. Inagaki, H. Nagai, Planta Med. 66 (2000) 25.

[15] R. Liu, M. Gao, G. Qiang, T. Zhang, X. Lan, J. Ying, G. Du, Neuroscience 162 (2009) 1232.

[16] A.A. Baskar, S. Ignacimuthu, G.P. Michae, K.S. Numair, Nutr. Cancer 63 (2011) 130.

[17] Y. Tian, L. Sun, X. Liu, B. Li, Q. Wang, J. Dong, Fitoterapia 81 (2010) 799.

[18] A.F.E. Rump, M. Schqssler, D. Acar, A. Cordes, M. Theisohn, R. Rosen, W. Klaus, U. Fricke, Gen. Pharmac. 25 (1994) 1137.

[19] K. Shimoia, H. Okadaa, M. Furugoria, T. Godaa, S. Takasea, M. Suzukib, Y. Harab, H. 
Yamamotoc, N. Kinaea, FEBS Lett. 438 (1998) 220.

[20] R. Domitrovic, H. Jakovac, C. Milin, B. Radosevic Stasic, Exp. Toxicol. Pathol. 61 (2009) 581.

[21] E. Zeinali, V. Haddadi-Asl, H. Roghani-Mamaqani, RSC Adv. 4 (2014) 31428.

[22] Y. Habibi, A. Goffin, N. Schiltz, E. Duquesne, P. Dubois, A. Dufresne, J. Mater. Chem. 18

(2008) 5002.

[23] G. Ränby, Acta Chem. Scand. 3 (1949) 649.

[24] S. Gilberto, B. Julien, D. Alain, Polymers 2 (2010) 728.

[25] C.C. Gary, S. Kristin, Nanoscale Res. Lett. 7 (2012) 192.

[26] S. Dong, H.J. Cho, Y.W. Lee, M. Roman, Biomacromolecules 15 (2014) 1560.

[27] H.Y. Youssef, L.A. Lucia, O.J. Rojas, Chem. Rev. 110 (2010) 3479.

[28] W. Bai, J. Holbery, K.C. Li, Cellulose 16 (2009) 455.

[29] D.E. Owens, N.A. Pappar, Int. J. Pharm. 307 (2006) 93.

[30] Y.Li, S.W. Wang, R. Huang, Z. Huang, B.F. Hu, W.F. Zheng, G. Yang, X.Y. Jiang, Biomacromolecules 16 (2015) 780.

[31] K. Rodriguez, S. Renneckar, P. Gatenholm, ACS Appl. Mater. Interfaces 3 (2011) 681.

[32] R.M.A. Domingues, M.E.Gomes, R.L. Reis, Biomacromolecules 15 (2014) 2327.

[33] C. Zhou, Q. Shi, W. Guo, L. Terrell, A.T. Qureshi, D.J. Hayes, Q. Wu, ACS Appl. Mater. Interfaces. 53 (2013) 3847.

[34] R. Rodriguez, C. Alvarez Lorenzo, A. Concheiro, J. Control. Release. 86 (2003) 253.

[35] S. Butun, F.G. Ince, H. Erdugan, N. Sahiner, Carbohyd. Polym. 86 (2011) 636.

[36] H. Wang, M. Roman, Biomacromolecules 12 (2011) 1585.

[37] A.N. Zelikin, ACS Nano. 4 (2010) 2494.

[38] C. Theron, A. Gallud, C. Carcel, M. Gary Bobo, M. Maynadier, M. Garcia, J. Lu, F. Tamanoi, J.I. Zink, M. Wong Chi Man, Chem. Eur. J. 20 (2014) 9372.

[39] J. Fang, Q. Zhou, X. L. Shi, B. H. Jiang, Carcinogenesis 28 (2007) 713.

[40] D. Klemm, F. Kramer, S. Moritz, T. Lindstrom, M. Ankerfors, D. Gray, A. Dorris, Angew. Chem. Int. Ed. 50 (2011) 5438.

[41] X.M. Dong, J.F. Revol, D.G. Gray, Cellulose 5 (1998) 19.

[42] G. Favaro, A. Clementi, V. Romani, J. Fluoresc. 17 (2007) 707.

[43] A. Amat, F.D. Angelis, A. Sgamellotti, S. Fantacci, Chem. Phys Lett. 462 (2008) 313. 